\title{
LOCAL SEMICONVEXITY OF KANTOROVICH POTENTIALS ON NON-COMPACT MANIFOLDS*
}

\author{
Alessio Figalli $^{1}$ And Nicola Gigli ${ }^{2}$
}

\begin{abstract}
We prove that any Kantorovich potential for the cost function $c=d^{2} / 2$ on a Riemannian manifold $(M, g)$ is locally semiconvex in the "region of interest", without any compactness assumption on $M$, nor any assumption on its curvature. Such a region of interest is of full $\mu$-measure as soon as the starting measure $\mu$ does not charge $n-1$-dimensional rectifiable sets.
\end{abstract}

Mathematics Subject Classification. 49Q20, 35J96.

Received July 4, 2009. Revised October 13, 2009.

Published online March 31, 2010.

\section{INTRODUCTION AND MAIN RESULT}

Let $(M, g)$ be an $n$-dimensional complete Riemannian manifold. We consider the Monge transport problem on $M$ with cost function $c(x, y)=\frac{d(x, y)^{2}}{2}$. This amounts to study the following problem: given two probability measures $\mu$ and $\nu$ on $M$, minimize

$$
\int_{M} c(x, S(x)) \mathrm{d} \mu(x)
$$

among all maps $S: M \rightarrow M$ such that $S_{\#} \mu=\nu$. The existence and uniqueness of an optimal transport map under the assumption that $\mu$ gives no mass to $(n-1)$-rectifiable sets and

$$
\inf _{S_{\#} \mu \nu} \int_{M} c(x, S(x)) \mathrm{d} \mu(x)<+\infty
$$

has been proved in $[4,5]$ (the result in the compact case was first proven in [8]). The strategy was the following: first of all, one consider the Kantorovich minimization problem

$$
\inf _{\gamma \in \Pi(\mu, \nu)} \int_{M \times M} c(x, y) \mathrm{d} \pi(x, y),
$$

Keywords and phrases. Kantorovich potential, optimal transport, regularity.

* N. Gigli was partially financed by KAM Faible, ANR-07-BLAN-0361.

${ }^{1}$ Centre de Mathématiques Laurent Schwartz, UMR 7640, École Polytechnique, 91128 Palaiseau, France.

figalli@math.polytechnique.fr

${ }^{2}$ University of Bordeaux, France. nicolagigli@googlemail.com 
where $\Pi(\mu, \nu)$ denotes the set of probability measures on $M \times M$ which have $\mu$ and $\nu$ as first and second marginal, respectively. Then, it is a well-known fact in optimal transport theory that an optimal $\pi$ exists, and it is contained in the $c$-subdifferential of a $c$-convex function $\psi$ (see [9], Thms. 4.1 and 5.10): this means that there exist two measurable functions $\phi: M \rightarrow \mathbb{R} \cup\{+\infty\}$ and $\psi: M \rightarrow \mathbb{R} \cup\{-\infty\}$ such that

$$
\phi(x)=\sup _{y \in M} \psi(y)-c(x, y), \quad \psi(y)=\inf _{x \in M} \phi(x)+c(x, y)
$$

and $\pi$ is concentrated on the set $\partial^{c} \phi:=\cup_{x \in M}\left(\{x\} \times \partial^{c} \phi(x)\right)$, where

$$
\partial^{c} \phi(x):=\{y \in M: \phi(x)=\psi(y)-c(x, y)\}
$$

This fact implies in particular that the set $\{\phi<+\infty\}$ has full $\mu$-measure.

Now, to complete the "classical" strategy in order to show existence and uniqueness of optimal maps, one needs to prove that $\phi$ is differentiable $\mu$-a.e. Indeed, this allows to show that $\partial^{c} \phi(x)$ is a singleton for $\mu$-a.e. $x$, which implies that the optimal plan $\pi$ is concentrated on the graph of a function $T$. Moreover, such a function can be characterized $\mu$-a.e. by the formula

$$
\left.\nabla_{x} c(x, y)\right|_{y=T(x)}=-\nabla \phi(x) \quad \Leftrightarrow \quad T(x)=\exp _{x}(\nabla \phi(x))
$$

(cf. [4], Thm. 4.3, or [7], Prop. 1.15, see also [8], Thm. 8).

However, in $[4,5]$ the authors could not exactly complete the program described above, since they were not able to show that the function $\phi$ (which is also called Kantorovich potential) is differential $\mu$-a.e. Indeed, since in the infimum appearing in (1.1) $y$ varies in a non-compact set, $\phi$ does not a priori inherit the local semiconvexity property of the functions $x \mapsto-\frac{d(x, y)^{2}}{2}$. Hence their strategy has been to prove a weaker statement, namely that $\phi$ is approximately differentiable $\mu$-a.e., which turns out to be enough for obtaining existence and uniqueness of the optimal map. Moreover, at least when $\mu$ is absolutely continuous with respect to the volume measure, (1.2) holds provided one replaces $\nabla$ by the approximate gradient $\tilde{\nabla}$ (see [4], Complement 3.4). The aim of this paper is to fill the gap on the regularity of $\phi$, showing that without any assumption on the manifolds nor on the measures $\mu$ and $\nu$, the potential $\phi$ is always locally semiconvex on the "region of interest". More precisely, we show the following:

Theorem 1.1. Let $\phi$ be a Kantorovich potential as above, set $D:=\{\phi<+\infty\}$ and let $\Omega$ be the interior of $D$. Then $\phi$ is locally semiconvex in $\Omega, \partial^{c} \phi(x)$ is non-empty for any $x$ in $\Omega$, and $\partial^{c} \phi$ is locally bounded in $\Omega$. Moreover, $D \backslash \Omega$ is $(n-1)$-rectifiable.

Once the above theorem is proved, the existence and uniqueness of an optimal map $T$ whenever $\mu$ does not charge $(n-1)$-rectifiable sets follows in a standard manner (see for instance [8], Lem. 7, or [4], Thm. 3.1). Moreover (1.2) holds $\mu$-a.e., which allows to deduce (thanks to the fact that a semiconcave function is twice differentiable vol-a.e.) that $T$ is differential $\mu$-a.e. provided $\mu \ll$ vol (see [3], Sect. 2, and [5], Prop. 3.4). This last fact is important in order to compute the Jacobian of $T$ and to obtain a change of variable formula (see [3], Cor. 4.7) which turns out to be useful for many different application in Riemannian geometry. We refer to [9] for a more complete presentation of the subject and more references.

The next section is devoted to the proof of the above theorem. At the end of the section, we will also discuss some possible extensions of the above result to the case $c=d^{p} / p(p>1)$, and to cost functions arising by minimizing a particular class of Tonelli Lagrangian. Moreover, we also make some general comments on how to deduce existence and uniqueness of optimal maps once the result is known in the compact case.

As a last comment, let us say that the approach we are going to describe is strongly inspired by a discussion made by Villani in [9], Chapter 10, where the author introduces the assumptions $\left(\mathbf{H}_{\left.\infty_{\mathbf{1}}\right)}\right.$ and $\left(\mathbf{H} \infty_{\mathbf{2}}\right)$, and he proves that if the cost function satisfies these assumptions, then a result closely related to ours holds (see [9], Thm. 10.24). The point is that it is unclear - at least to us - whether the squared distance satisfies those 
assumptions or not (in particular, the problem is in checking $\left(\mathbf{H} \infty_{\mathbf{2}}\right)$ ). Still, at least in the case $c=d^{p} / p$ or for costs coming from some special Tonelli Lagrangians, it is possible to conclude. We invite the reader to compare the proof of our result with the one of [9], Theorem 10.24.

Notice also that an approach similar to ours already appeared in [6], Appendix C.

\section{Proof of the MAIN TheOREM}

Recall that $D:=\{\phi<+\infty\}$ and $\Omega$ is the interior of $D$. We divide the proof in three steps.

\section{Step 1: $\phi$ is locally bounded in $\Omega$.}

Since $\phi$ is defined by a supremum of continuous functions (see (1.1)), the fact that $\phi$ is locally bounded from below is immediate. Hence we only need to prove the bound from above.

We argue by contradiction, and we assume the existence of a sequence $x_{n} \rightarrow \bar{x} \in \Omega$ such that $\phi\left(x_{n}\right) \rightarrow+\infty$. For every $n \in \mathbb{N}$, let us choose $y_{n} \in M$ a point such that

$$
\phi\left(x_{n}\right) \leq \psi\left(y_{n}\right)-c\left(x_{n}, y_{n}\right)+1 .
$$

In particular, as $c=\frac{d^{2}}{2} \geq 0$, we have $\psi\left(y_{n}\right) \rightarrow+\infty$ too. Hence, since

$$
\mathbb{R} \ni \phi(\bar{x}) \geq \psi\left(y_{n}\right)-c\left(x, y_{n}\right),
$$

we deduce that $c\left(x, y_{n}\right) \rightarrow+\infty$, which further implies

$$
c\left(x_{n}, y_{n}\right)=\frac{d\left(x_{n}, y_{n}\right)^{2}}{2} \rightarrow+\infty .
$$

Now, let $\gamma_{n}:\left[0, d\left(x_{n}, y_{n}\right)\right] \rightarrow M$ be a minimizing geodesic parameterized by arc-length connecting $x_{n}$ to $y_{n}$. Since $d\left(x_{n}, y_{n}\right) \rightarrow+\infty$, any geodesic $\gamma_{n}$ is defined at least on an interval $[0, \ell]$, for some $\ell>0$. Let us define the following set:

$$
C_{n}:=\left\{x \in M: \text { there exists } t \in[0, \ell] \text { s.t. } d\left(x, \gamma_{n}(t)\right) \leq t / 2\right\} .
$$

(Observe that in an Euclidean space $C_{n}$ would just be a cone with height $\ell$ and basis of radius $\ell / 2$.) We claim that

$$
\inf _{C_{n}} \phi \rightarrow+\infty \quad \text { as } n \rightarrow+\infty .
$$

Indeed, if $d\left(x, \gamma_{n}(t)\right) \leq t / 2$ for some $t \in[0, \ell]$, thanks to the triangle inequality and (2.1) we have

$$
\begin{aligned}
\phi(x) & \geq \psi\left(y_{n}\right)-\frac{d\left(x, y_{n}\right)^{2}}{2} \geq \psi\left(y_{n}\right)-\frac{\left[d\left(\gamma_{n}(t), y_{n}\right)+d\left(x, \gamma_{n}(t)\right)\right]^{2}}{2} \\
& \geq \psi\left(y_{n}\right)-\frac{\left[d\left(\gamma_{n}(t), y_{n}\right)+t / 2\right]^{2}}{2}=\psi\left(y_{n}\right)-\frac{\left[d\left(x_{n}, y_{n}\right)-t / 2\right]^{2}}{2} \\
& \geq \psi\left(y_{n}\right)-\frac{d\left(x_{n}, y_{n}\right)^{2}}{2}+d\left(x_{n}, y_{n}\right) \frac{t}{2}-\frac{\ell^{2}}{8} \\
& \geq \phi\left(x_{n}\right)-1+d\left(x_{n}, y_{n}\right) \frac{t}{2}-\frac{\ell^{2}}{8},
\end{aligned}
$$

where at the second line we used the identity $d\left(\gamma_{n}(t), y_{n}\right)=d\left(x_{n}, y_{n}\right)-t$. Thanks to the above inequality, we obtain that

$$
\inf _{C_{n}} \phi \geq \phi\left(x_{n}\right)-1-\frac{\ell^{2}}{8},
$$


which proves the claim. Now, letting $n \rightarrow+\infty$ and assuming $\ell$ sufficiently small (say, $1 / 10$ of the minimal injectivity radius in a neighborhood of $\bar{x}$ ), it is easy to see by a simple compactness argument that the following holds: let $v \in T_{\bar{x}} M$ be a limit point for $\dot{\gamma}_{n}(0) \in T_{x_{n}} M$ (in some chart around $\bar{x}$ ), and set

$$
C_{\infty}:=\left\{x \in M: \text { there exists } t \in[0, \ell] \text { s.t. } d\left(x, \exp _{\bar{x}}(t v)\right) \leq t / 2\right\} .
$$

Then, up to subsequences, $C_{n} \rightarrow C_{\infty}$ in the Hausdorff distance, and $\phi \equiv+\infty$ in the interior of $C_{\infty}$. Since $\bar{x} \in C_{\infty}$, this contradicts the fact that $\bar{x} \in \Omega$, and concludes the proof.

Step 2: $D \backslash \Omega$ is $(n-1)$-rectifiable.

This fact is a simple consequence of the proof of Step 1: assume that $\bar{x} \in D \backslash \Omega$, and let $\left\{x_{n}\right\} \subset D^{c}$ be a sequence converging to $\bar{x}$. Then, choosing a sequence $\left\{y_{n}\right\} \subset M$ such that

$$
\psi\left(y_{n}\right)-c\left(x_{n}, y_{n}\right) \geq n
$$

(this can always be done as $\phi\left(x_{n}\right)=+\infty$ ), we deduce that

$$
\inf _{C_{n}} \phi \geq n-1
$$

(compare with (2.3)), so that $\phi \equiv+\infty$ in the interior of a "cone" $C_{\infty}$ with vertex at $\bar{x}$, height $\ell$ and width $\ell / 2$ (see (2.4)). Hence we have proved that at every point $\bar{x} \in D \backslash \Omega$ there exists an open cone of fixed height and width, with vertex at $\bar{x}$, which is contained outside $D$, and thus outside $D \backslash \Omega$. Then, it is a well-known result in geometric measure theory that $D \backslash \Omega$ is $(n-1)$-rectifiable, i.e. contained in a countable union of Lipschitz surfaces (see for instance the proof of [1], Thm. 2.61, or the one of [9], Thm. 10.48).

Step 3: $\partial^{c} \phi(x)$ is non-empty and bounded as $x$ varies in a compact subset of $\Omega$. In particular, $\phi$ is locally semiconvex in $\Omega$.

Let $K \subset \subset \Omega$, take $\bar{x} \in K$, and let $y \in M$ be such that

$$
\phi(\bar{x}) \leq \psi(y)-c(\bar{x}, y)+1 .
$$

We claim that $d(\bar{x}, y)$ is uniformly bounded, independently of $y$. Indeed, assuming without loss of generality $d(\bar{x}, y) \geq 1$, as in the proof of Step 1 we can consider the point $\gamma(\ell)$ on the (unit speed) geodesic from $\bar{x}$ to $\bar{y}$, where $\ell \leq \operatorname{dist}\left(K, \Omega^{c}\right) / 2$. Then, by $(2.2)$ we get

$$
\phi(\gamma(\ell)) \geq \phi(\bar{x})-1+d(\bar{x}, y) \frac{\ell}{2}-\frac{\ell^{2}}{8} .
$$

Since by Step $1 \phi$ is uniformly bounded on the set

$$
K_{\ell}:=\{x \in M: \operatorname{dist}(x, K) \leq \ell\} \subset \subset \Omega,
$$

the claim follows.

The proof of Step 3 is now easy: thanks to (1.1) the function $\psi(y)$ is upper semicontinuous. Hence, if $\bar{x} \in K$ and $\left\{y_{k}\right\} \subset M$ is a maximizing sequence for $\phi$, in the sense that

$$
\phi(\bar{x}) \leq \psi\left(y_{k}\right)-c\left(\bar{x}, y_{k}\right)+\frac{1}{k},
$$

then by compactness (recall that $d\left(\bar{x}, y_{k}\right)$ is uniformly bounded) there exists a point $\bar{y}$ such that

$$
\phi(\bar{x}) \leq \psi(\bar{y})-c(\bar{x}, \bar{y}) .
$$


Since the opposite inequality is always true, we obtain that equality holds and $\bar{y} \in \partial^{c} \phi(\bar{x})$. Moreover $\operatorname{dist}(K, \bar{y}) \leq$ $C_{0}$ for some constant $C_{0}$ depending only on $K$. Hence

$$
\phi(x)=\sup _{y \in M, \operatorname{dist}(K, \bar{y}) \leq C_{0}} \psi(y)-c(x, y) \quad \forall x \in K,
$$

and the semiconvexity of $\phi$ in the interior of $K$ follows. This concludes the proof of the main theorem.

Remark 2.1 (the case $\left.c=d^{p} / p\right)$. The above result can be easily extended to the case $c(x, y)=\frac{d(x, y)^{p}}{p}, p>1$. Indeed the only difference arises in the proof of (2.2), where by using the inequality $(a-b)^{p} \leq a^{p}-p a^{p-1} b$ for any $0 \leq b \leq a$ we get

$$
\phi(x) \leq \phi\left(x_{n}\right)-1+d\left(x_{n}, y_{n}\right)^{p-1} \frac{t}{2} \quad \forall x \in C_{n} .
$$

Once one has the above inequality, the rest of the proof follows with no changes. Let us however point out that, since $x \mapsto d^{p}(x, y) / p$ is not semiconcave in the classical sense (i.e. distributional locally second derivatives bounded from above) but only with a modulus of semiconcavity $\omega(t)=t^{p-1}$ (see [4], Appendices A and B), the potential function will only be $\omega$-semiconvex, which is however enough for the potential to be differentiable out of a countably $(n-1)$-rectifiable set.

Remark 2.2 (costs induced by Lagrangian). A variant of the above argument still works when the cost function arises by minimizing a Lagrangian which behaves like $\theta\left(|v|_{x}\right)$, where $\theta:[0,+\infty) \rightarrow[0,+\infty)$ is convex, superlinear, and $\theta(0)=0$ :

$$
c(x, y)=\inf _{\gamma(0)=x, \gamma(1)=y} \int_{0}^{1} L(\gamma(t), \dot{\gamma}(t)) \mathrm{d} t
$$

when $L: T M \rightarrow \mathbb{R}$ is a Lagrangian satisfying:

(a) $L$ is $C^{2}$;

(b) for every $(x, v) \in T M, \frac{\partial^{2} L}{\partial v^{2}}(x, v)$ is positive definite on $T_{x} M$;

(c) there exist a convex superlinear function $\theta: \mathbb{R}^{+} \rightarrow \mathbb{R}^{+}$, with $\theta(0)=0$, and $A_{1}, A_{2}, A_{3}$ positive constants, such that

$$
\theta\left(|v|_{x}\right)-A_{1} \leq L(x, v) \leq A_{2} \theta\left(|v|_{x}\right)+A_{3}, \quad \forall(x, v) \in T M .
$$

Indeed, the only main difference consists in the choice of the cone $C_{n}$ and the proof of (2.2): if $\bar{x} \in M$, and $\left(x_{n}\right),\left(y_{n}\right)$ are like in Step 1, we consider $\gamma_{n}:[0,1] \rightarrow M$ to be a minimizer for $L$ going from $x_{n}$ to $y_{n}$, that is

$$
c\left(x_{n}, y_{n}\right)=\int_{0}^{1} L\left(\gamma_{n}(t), \dot{\gamma}_{n}(t)\right) \mathrm{d} t, \quad \gamma_{n}(0)=x_{n}, \gamma_{n}(1)=y_{n}
$$

and we set

$$
C_{n}:=\left\{x \in M \text { : there exists } t \in[0,1] \text { s.t. } d\left(x, \gamma_{n}(t)\right) \leq \eta d\left(x_{n}, \gamma_{n}(t)\right) \text {, with } d\left(x_{n}, \gamma_{n}(t)\right) \leq \ell\right\},
$$

where $\eta, \ell>0$ are small constants to be chosen. Let now $x \in M$ be such that $d\left(x, \gamma_{n}(t)\right) \leq \eta t$ for some $t \in[0, \ell]$, and take $\sigma:[0, t] \rightarrow M$ a constant-speed minimizing geodesic connecting $\gamma_{n}(t)$ to $x$. Then $|\dot{\sigma}(\tau)|_{\sigma(\tau)}=$ $d\left(x_{n}, \gamma_{n}(t)\right) / t \leq \eta d\left(x, \gamma_{n}(t)\right) / t$, and thanks to the above assumptions on $L$ we get

$$
\begin{aligned}
c\left(x, y_{n}\right)-c\left(x_{n}, y_{n}\right) & \leq \int_{0}^{t} L(\sigma(\tau), \dot{\sigma}(\tau)) \mathrm{d} \tau-\int_{0}^{t} L\left(\gamma_{n}(\tau), \dot{\gamma}_{n}(\tau)\right) \mathrm{d} \tau \\
& \leq \int_{0}^{t} A_{2} \theta\left(|\dot{\sigma}(\tau)|_{\sigma(\tau)}\right) \mathrm{d} \tau-\int_{0}^{t} \theta\left(\left|\dot{\gamma}_{n}(\tau)\right|_{\gamma_{n}(\tau)}\right) \mathrm{d} \tau+\left(A_{1}+A_{3}\right) t \\
& \leq t\left[A_{2} \theta\left(\eta \frac{d\left(x, \gamma_{n}(t)\right)}{t}\right)-\theta\left(\frac{d\left(x, \gamma_{n}(t)\right)}{t}\right)+\left(A_{1}+A_{3}\right)\right],
\end{aligned}
$$


where at the last step we used Jensen's inequality to estimate the second term. We now observe that, since $\theta(0)=0$ and $\theta$ is convex, $\theta(\eta s) \leq \eta \theta(s)$ for all $s \geq 0$. Hence, if we choose $\eta=1 /\left(2 A_{2}\right)$ we have

$$
c\left(x_{n}, y_{n}\right)-c\left(x, y_{n}\right) \geq \frac{t}{2} \theta\left(\frac{d\left(x, \gamma_{n}(t)\right)}{t}\right)-\left(A_{1}+A_{3}\right) t
$$

and arguing as in (2.2) we obtain

$$
\phi(x) \geq \phi\left(x_{n}\right)-1+\frac{t}{2} \theta\left(\frac{d\left(x, \gamma_{n}(t)\right)}{t}\right)-\left(A_{1}+A_{3}\right) t .
$$

This estimate allows to prove that if $\phi\left(x_{n}\right) \rightarrow+\infty$, then there exists a cone with (locally) fixed height and width, and with vertex at $\bar{x}$, such that $\phi \equiv+\infty$ inside this cone. This allows to prove Steps 1 and 2 . To get Step 3, we have to show that, if

$$
\phi(\bar{x}) \leq \psi(y)-c(\bar{x}, y)+1
$$

then $d(x, y)$ is uniformly bounded. The proof of this fact is analogous of the one above, although a bit more involved: thanks to the above assumptions, the Hamiltonian $H(x, p)$ behaves like $\theta^{*}\left(|p|_{x}\right)$, where $\theta^{*}$ is the Legendre transform of $\theta$. Hence, by exploiting the conservation of the Energy, as in proof of [4], Proposition B.17, one can show that $\left|\dot{\gamma}\left(t_{0}\right)\right|$ at some $t_{0} \in[0,1]$ controls $\max _{t \in[0,1]}|\dot{\gamma}(t)|$ if $\gamma$ is a minimizer. In this way, one can still prove $d(x, y)$ has to be uniformly bounded, and the proof of Step 3 follows easily. (We leave the details to the interested reader.)

Remark 2.3 (compact vs. non-compact). Let us point out that, if one is simply interested in knowing that the optimal transport map exists and is unique, without having any information on its structure (namely, that is induced by a Kantorovich potential through the first formula in (1.2)), then the argument for passing from the compact to the non-compact case is pretty simple (what we are going to say is not new - e.g. the argument was used already in [2], Thm. 6.2.10 - still we believe it is worth to repeat it here). Assume indeed that existence and uniqueness of optimal maps is known whenever $\mu$ and $\nu$ are compactly supported (together with some suitable assumptions on $\mu$ ). Then, in the general case, one simply takes an optimal plan $\pi$ and consider its restriction to $K_{n} \times K_{n}$, where $\left\{K_{n}\right\}$ is an increasing sequence of compact sets such that $\cup_{n} K_{n}=M$. If $\mu_{n}$ and $\nu_{n}$ denote the first and second marginal of $\pi_{n}:=\frac{\pi L_{\left(K_{n} \times K_{n}\right)}}{\pi\left(K_{n} \times K_{n}\right)}$ respectively, then it is well-known that $\pi_{n}$ is an optimal transport plan between them (see for instance [9], Thm. 4.6). Hence any $\pi_{n}$ is concentrated on a graph, and letting $n \rightarrow+\infty$ also $\pi$ has to be concentrated on a graph. This proves the existence. Moreover the fact that any optimal plan is concentrated on a graph immediately implies uniqueness, as a linear combination of optimal plans is still optimal.

\section{REFERENCES}

[1] L. Ambrosio, N. Fusco and D. Pallara, Functions of bounded variation and free discontinuity problems, Oxford Mathematical Monographs. The Clarendon Press, Oxford University Press, New York (2000).

[2] L. Ambrosio, N. Gigli and G. Savaré, Gradient flows in metric spaces and in spaces of probability measures, Lectures in Mathematics ETH Zürich. Birkhäuser Verlag, Basel (2005).

[3] D. Cordero-Erasquin, R.J. McCann and M. Schmuckenschlager, A Riemannian interpolation inequality à la Borell, Brascamp and Lieb. Invent. Math. 146 (2001) 219-257.

[4] A. Fathi and A. Figalli, Optimal transportation on non-compact manifolds. Israel J. Math. (to appear).

[5] A. Figalli, Existence, uniqueness, and regularity of optimal transport maps. SIAM J. Math. Anal. 39 (2007) 126-137.

[6] W. Gangbo and R.J. McCann, The geometry of optimal transportation. Acta Math. 177 (1996) 113-161.

[7] N. Gigli, Second order analysis on $\left(\mathscr{P}_{2}(M), W_{2}\right)$. Memoirs of the AMS (to appear), available at http://cvgmt.sns.it/cgi/ get.cgi/papers/gig09/.

[8] R.J. McCann, Polar factorization of maps on Riemannian manifolds. Geom. Funct. Anal. 11 (2001) 589-608.

[9] C. Villani, Optimal transport, old and new, Grundlehren des mathematischen Wissenschaften [Fundamental Principles of Mathematical Sciences] 338. Springer-Verlag, Berlin-New York (2009). 